\title{
Rede de Sensores Sem Fio para Monitoramento de Variáveis de Ambiente
}

\author{
Lucas José da Cunha \\ Universidade do Vale do Itajaí - UNIVALI \\ lucas_cunha@edu.univali.br
}

\begin{abstract}
This research has the objective of implementing a sensors network for monitoring variable of environments using an IoT(Internet of Things) technology focused on low energy consumption and long range. The idea is to implement this network using LoRa technology and LoRaWaN protocol. For a better utilization, it will be used and tested different LoRa parameters to communicate the nodes aiming show the impacts of these parameters changes in long range, with consistent data and low power consumption.
\end{abstract}

\section{KEYWORDS}

IoT, Rede de sensores, Lora, Lorawan, Baixo custo

\author{
Paulo Roberto Oliveira Valim \\ Universidade do Vale do Itajaí - UNIVALI \\ pvalim@univali.br
}

Table 1: Visão geral das tecnologias LPWAN: SigFox, LoRa e NB-IoT[2]

\begin{tabular}{lccc}
\hline & SigFox & LoRaWAN & NB-IoT \\
\hline Larg de banda & $100 \mathrm{~Hz}$ & 250kHz e 125kHz & $200 \mathrm{kHz}$ \\
Tx. máx. de dados & $100 \mathrm{bps}$ & $50 \mathrm{kbps}$ & $200 \mathrm{kbps}$ \\
Max. de msg./dia & 140 & Ilimitado & Ilimitado \\
Tam. máx. de msg. & 12 bytes & 243 bytes & 1600 bytes \\
Alcance & 10km à 40km & $5 \mathrm{~km}$ à $20 \mathrm{~km}$ & $1 \mathrm{~km}$ à $10 \mathrm{~km}$ \\
Imun. a Interf. & Muito Alto & Muito Alto & Baixo \\
Tx. de dados adapt. & Não & Sim & Não \\
Permite rede priv. & Não & Sim & Não \\
\hline
\end{tabular}

A rede SigFox [3] é uma rede narrow-band ou ultra narrowband. A empresa Sigfox e seus 2 operadores detêm o controle da tecnologia, servidores, abrindo espaço para outras entidades desenvolverem os dispositivos periféricos(end-points). Empresas como a ST, Silicon Labs e Microchip fabricam rádios compatíveis com a tecnologia. Uma empresa interessada em desenvolver um produto baseado em Sigfox deve desenvolver um hardware com um módulo sub-GHz compatível com a tecnologia e contatar o operador da região para informações sobre custo e pacotes de dados. O organismo de normas internacionais 3GPP concluiu a normatização do NB-IoT [4] em meados de 2016. Este padrão usa frequências licenciadas, as mesmas usadas por algumas frequências do $4 \mathrm{G}$, permitindo que algumas estações sejam atualizadas e compartilhem sua infraestrutura. A rede LoRaWAN [5] é uma solução sem fio sub-GHz em frequência não licenciada que endereça demandas para conexão entre dispositivos para aplicações de baixo consumo, longa distância (em alguns locais é possível conseguir $15 \mathrm{~km}$ ) e baixo custo de infraestrutura, considerando-se o grande número de nós. Diferente da SigFox e NB-IoT, LoRaWAN oferece a possibilidade de desenvolvimento de redes privadas e facilidade de integração com plataformas de diferentes fabricantes e devido a isto chamou a atenção de pesquisadores desde seu surgimento no mercado, com mais de 7000 publicações listadas pela base de dados Google Scholar (2020, GOOGLE SCHOLAR) contendo a palavra chave "LoRaWAN" e 262 publicações na base de dados IEEE Explore (IEEE EXPLORER, 2019).

Este trabalho de pesquisa tem como proposta o desenvolvimento de uma rede de sensores utilizando tecnologia LoRa, onde os sensores irão capturar variáveis de ambiente, tais como pressão atmosférica, temperatura, luminosidade e/ou etc. em diferentes pontos espalhados pelo Campus da UNIVALI Itajaí, e sem utilizar da rede de comunicação da universidade. 


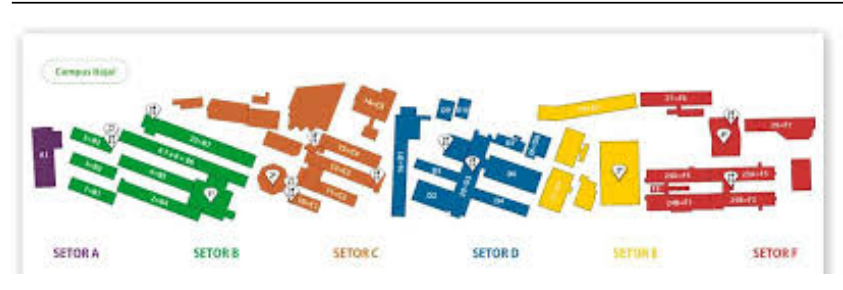

Figure 1: Campus da UNIVALI - Itajaí.

\section{DESENVOLVIMENTO}

Para melhor entendimento da estrutura utilizada no projeto é necessário frisar a tecnologia utilizada. O nome LoRa vem do inglês Long Range (Longo Alcance em português) e trata-se de uma tecnologia proprietária pertencente à empresa Semtech (SEMTECH, 2015). LoRa é uma técnica de modulação de espectro de dispersão derivada da tecnologia "Chirp Spread Spectrum" (Espalhamento de espectro por chirp em português). A modulação Chirp Spread Spectrum (CSS) mantém as características de baixa potência da modulação FSK. É uma técnica de espectro de dispersão que usa pulsos de chirp modulados em frequência linear de banda larga para codificar informações. Esta modulação define que cada bit é espalhado por um "chipping fator", e o número de chips por bit é chamado de Fator de Espalhamento (Spreading factor - SF). Pequenos fatores de espalhamento fornecem alta taxa de dados e exigem menos tempo no ar, e grandes fatores fornecem baixas taxas de dados e exigem mais tempo no ar. A estrutura LoRaWAN é formada por diversos componentes como ilustra a Figura 2.

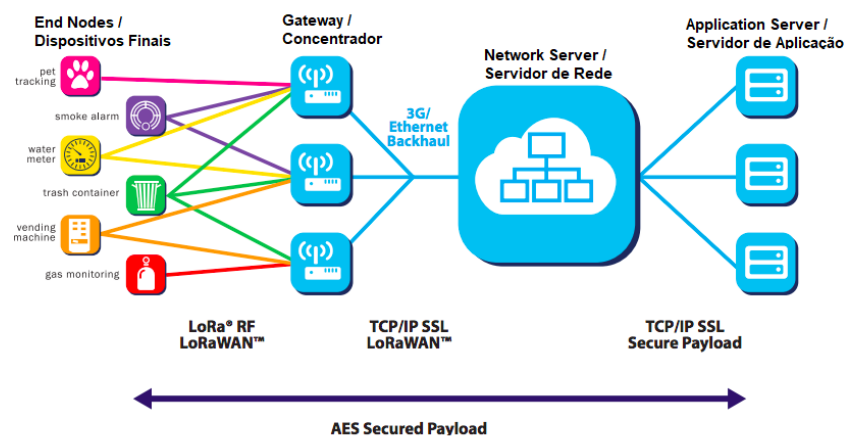

Figure 2: Estrutura da arquitetura de rede LoRa ${ }^{\circledR}$.

Os Dispositivos Finais/Periféricos correspondem aos módulos microcontroladores com sensores de temperatura, pressão, de movimento, atuador do tipo liga/desliga, entre outros. Estes Dispositivos Finais possuem um transceptor LoRa que se comunicam com os Gateways sem fio usando a modulação LoRa. Os Gateways, por sua vez, recebem "frames" brutos LoRaWAN de Dispositivos Finais e os encaminham para um servidor de rede tipicamente Ethernet, $3 \mathrm{G} / 4 \mathrm{G}$ ou Wi-Fi. Um Gateway pode receber dados de milhares de sensores e dependendo da topologia do local, pode cobrir um raio de 2,5 a 15 $\mathrm{km}$. Os Servidores de Rede são responsáveis pelo gerenciamento das informações enviadas pelos Gateways. Os Servidores de Aplicações são programas específicos que recebem (via requisição ou de forma automática) os pacotes dos Servidores de Rede e de acordo com a informação executam uma ou mais ações específicas. É importante enfatizar que “... a estrutura LoRaWAN define dois tipos bem diferenciados de camada, a camada PHY, definida pela modulação LoRa, e a camada MAC, definida pelo protocolo LoRaWAN." [6]. Sendo o objetivo principal do projeto explorar a modulação LoRa e sua camada física (PHY), descartando incialmente a parte dos servidores de rede e servidores de aplicação onde são os principais atuadores do protocolo LoRaWAN.

Uma dúvida natural para a maioria dos estudantes e profissionais que desejam se aprofundar nas diferentes tecnologias para IoT é a escolha do hardware a ser usado e qual o seu custo associado. Para este projeto o desenvolvimento foi baseado em cima de testes com módulos que são transceptores (chips) LoRa, sendo eles: Arduino UNO conectado um shield com chip RFM95W [7] da HopeRF, e o ESP32 com chip SX1276 [8] da SEMTECH. Para utilizar como concentrador de sinal (gateway) da rede foi utilizado o Dragino LG01P Gateway [9]. Como mencionado acima, foi necessário adquirir módulos com frequência licenciada no Brasil, que neste caso foi escolhido módulos de $915 \mathrm{MHz}$.

Para o desenvolvimento de códigos foi utilizado a IDE Visual Studio Code com o plugin PlatformIO e a IDE do Arduino sendo implementado em $\mathrm{C}++$ com o pacote de bibliotecas RadioHead [10] que é completa para comunicação de radiofrequência com foco em sistemas embarcados. Dentro deste pacote de bibliotecas existe uma biblioteca específica que contém os métodos para alterar os parâmetros LoRa, que é a biblioteca "RH_RF95.h".

\section{TESTES E RESULTADOS}

Para início dos testes, deve-se apresentar um trabalho relacionado a este onde foram feitos testes utilizando alguns parâmetros LoRa já definidos pelo autor Marco Cattani et al. [11] sendo eles nomeados de "Setting ID". Tendo em vista um cenário com grande tráfego de comunicação de dados em diferentes frequências e contando com um local com diversos obstáculos físicos, os testes tendem a ser mais sólidos e sendo analisados no pior caso possível. O objetivo da fase de testes era validar três importantes requisitos, sendo eles: alcance de comunicação entre end node e gateway, garantir a integridade dos dados, e consumo energético reduzido ao mínimo.

Em cada configuração de parâmetros (Setting ID) utilizada foram alterados parâmetros da camada PHY (física) sendo eles:

- SF: Spreading Factor (Fator de espalhamento).

- CR: Coding Rate (Taxa de codificação).

- BW: Bandwidth (Largura de banda).

- BR: Bit rate (Taxa de bits).

A etapa de testes foi dividida em duas partes, a primeira parte de medição de distância e integridade de dados e a segunda parte sendo a de consumo energético.

\subsection{Distância e Integridade}

O roteiro de testes da primeira parte ocoreu da seguinte forma: Foram estabelecidos certos pontos fixos na universidade, onde em cada ponto fixo eram deixados os módulos emitindo pacotes de 256 bytes (tamanho máximo) num intervalo de 10 s e sendo alterado os Setting ID's com o intuito de conseguir maior distância e integridade dos dados enviados. 
Table 2: Módulo SX1276

\begin{tabular}{ccc}
\hline Distância & Taxa de recepção de pacotes & Setting ID \\
\hline $50 \mathrm{~m}$ & $99,2 \%$ & 6 \\
$150 \mathrm{~m}$ & $74,5 \%$ & 16 \\
$200 \mathrm{~m}$ & $14,7 \%$ & 11 \\
\hline
\end{tabular}

Table 3: Módulo RFM95W

\begin{tabular}{ccc}
\hline Distância & Taxa de recepção de pacotes & Setting ID \\
\hline $50 \mathrm{~m}$ & $100 \%$ & 7 \\
$150 \mathrm{~m}$ & $100 \%$ & 11 \\
$200 \mathrm{~m}$ & $9,41 \%$ & 16 \\
\hline
\end{tabular}

Avaliando as configurações estabelecidas para o alcance de distância máximo foi observado que foram utilizados os parâmetros que melhor se encaixam quando se trata de longa distância e envio de poucos bytes. Tendo em conta que o Setting ID 16 tem um fator de espalhamento alto, taxa de bits pequena, taxa de codificação alta e o parâmetro "powerTX" que define quanto de energia separada para o envio definido ao máximo, o esperado era uma distância maior com uma maior taxa de recepção de dados.

\subsection{Consumo de Energia}

$\mathrm{O}$ teste de consumo foi realizado da seguinte forma: $\mathrm{O}$ módulo emissor e o gateway se comunicavam-se de 1 metro de disância e foi utilizado um multímetro digital para mensuração. Antes de mostrar o resultado é importante ressaltar na alteração feita no código para melhor aproveitamento de energia dos módulos, sendo explorado o modo Deep Sleep (Sono Profundo) que os microcontroladores possuem, onde o microcontrolador desliga alguns recursos não utilizados em seu hardware como por exemplo: desabilitar o CPU, parte da RAM, módulo de WiFi e outros recursos inúteis ao nosso caso. Tanto o Arduino UNO quanto o ESP32 possuem esse modo, diferenciando-se apenas alguns recursos desligados. Os testes foram realizados com diferentes Setting ID’s, porém não foi observada nenhuma alteração de consumo, já que o único parâmetro que poderia alterar seu consumo seria o "powerTX" que foi inicialmente definido ao máximo (no código o máximo é $20 \mathrm{dBm}$ ) para garantir integridade dos dados.

Table 4: Teste de consumo energético em diferentes modos.

\begin{tabular}{lcc}
\hline & Deep Sleep & Emitindo Dados \\
\hline ESP32 + SX1276 & $10,56 \mathrm{~mA}$ & $61,20 \mathrm{~mA}$ \\
UNO + RFM95W & $16,19 \mathrm{~mA}$ & $27,19 \mathrm{~mA}$ \\
\hline
\end{tabular}

\section{CONSIDERAÇÕES FINAIS}

Se tratando dos resultados dos testes é nítido a diferença entre o especulado e o adquirido, sendo relevado alguns motivos mostrando que foram testes realizados em cenários difíceis de conseguir ótimos resultados. Algumas possibilidades são discutidas para o recepetor não receber as mensagens de uma distância maior e não obter menor consumo, tais como:

- O Gateway estava a uma distância muito curta do chão, o ideal é posiciona-lo em um local alto.

- As antenas dos módulos utilizados não são das melhores o que pode provocar impacto na transmissão.

- O tamanho da mensagem enviada impacta na distância, visando que sempre foi exposto uma mensagem ao máximo de 256 bytes.

- Diversos obstáculos na universidade, como paredes e circulação de pessoas.

- A escolha de um microcontrolador com menor consumo energético.

O objetivo de realizar uma rede de sensores sem fio e sem utilizar a rede da universidade foi concretizado, ressaltando que o objetivo não eram obter amostras das variáveis de ambiente em si, mas sim explorar a tecnologia e a arquitetura LoRa. Apesar de ter trabalhos relacionados onde testaram também a distância como fator, a parte de conservação de energia não foi tão explorada em outros trabalhos, sendo um ponto importante para diferenciação deste artigo.

\section{ACKNOWLEDGMENTS}

Gostaria de agradecer encarecidamente ao orientador do projeto Paulo Valim por me selecionar para fazer parte desta pesquisa, pela ajuda em todo cronograma e pela disponibilização dos equipamentos. Agradeço aos membros do Laboratório de Redes de Computadores onde foi realizada a pesquisa que sempre pudemos trocar ideias e incentivar a pesquisa, e a Secretaria da Educação do Estado de Santa Catarina por proporcionar a bolsa de Pesquisa do Artigo 170 .

\section{REFERENCES}

[1] Cisco. Internet of things at a glance, 2016. URL https:/www.cisco.com/c/dam/ en/us/products/collateral/se/internet-of-things/at-a-glance-c45-731471.pdf.

[2] Kais Mekki, Eddy Bajic, Frederic Chaxel, and Fernand Meyer. A comparative study of lpwan technologies for large-scale iot deployment. ICT express, 5(1):1-7, 2019.

[3] Sigfox. Sigfox technology, 2020. URL https://www.sigfox.com/en/what-sigfox/ technology.

[4] NB-IoT. Narrowband iot, 2020. URL https://www.3gpp.org/component/ itpgooglesearch/search?gsquery=NarrowBand+IOT.

[5] Semtech. What is lora?, 2020. URL https://www.semtech.com/lora/what-is-lora.

[6] Ramon Sanchez-Iborra, Jesus Sanchez-Gomez, Juan Ballesta-Viñas, Maria-Dolores Cano, and Antonio F Skarmeta. Performance evaluation of lora considering scenario conditions. Sensors, 18(3):772, 2018.

[7] RFM95/96/97/98(W) - Low Power Long Range Transceiver Module. HOPE MICROELECTRONICS, 2016. Rev. 1.

[8] SX1276/77/78/79 - $137 \mathrm{MHz}$ to $1020 \mathrm{MHz}$ Low Power Long Range Transceiver. Semtech Corporation, 1 2019. Rev. 6.

[9] Open Source LoRa WiFi Gateway. Dragino Technology Co., 8 2019. Rev. 1.

[10] Mike McCauley. Radiohead packet radio library for embedded microprocessors, October 2019. URL https://www.airspayce.com/mikem/arduino/RadioHead/.

[11] Marco Cattani, Carlo Alberto Boano, and Kay Römer. An experimental evaluation of the reliability of lora long-range low-power wireless communication. fournal of Sensor and Actuator Networks, 6(2):7, 2017. 